Article

\title{
High Order Two-Derivative Runge-Kutta Methods with Optimized Dispersion and Dissipation Error
}

\author{
Theodoros Monovasilis ${ }^{1, *,+}$ (D) and Zacharoula Kalogiratou ${ }^{2, *,+}$ (D) \\ 1 Department of Economics, University of Western Macedonia, 50100 Kozani, Greece \\ 2 Department of Informatics, University of Western Macedonia, 50100 Kozani, Greece \\ * Correspondence: tmonovasilis@uowm.gr (T.M.); zkalogiratou@uowm.gr (Z.K.) \\ + These authors contributed equally to this work.
}

Citation: Monovasilis, T.;

Kalogiratou, Z. High Order

Two-Derivative Runge-Kutta Methods with Optimized Dispersion and Dissipation Error. Mathematics 2021, 9, 232. https://doi.org/ $10.3390 /$ math 9030232

Received: 4 November 2020 Accepted: 18 January 2021 Published: 25 January 2021

Publisher's Note: MDPI stays neutral with regard to jurisdictional clai$\mathrm{ms}$ in published maps and institutional affiliations.

Copyright: (C) 2021 by the authors. Licensee MDPI, Basel, Switzerland. This article is an open access article distributed under the terms and conditions of the Creative Commons Attribution (CC BY) license (https:// creativecommons.org/licenses/by/ $4.0 /)$.

\begin{abstract}
In this work we consider explicit Two-derivative Runge-Kutta methods of a specific type where the function $f$ is evaluated only once at each step. New 7th order methods are presented with minimized dispersion and dissipation error. These are two methods with constant coefficients with 5 and 6 stages. Also, a modified phase-fitted, amplification-fitted method with frequency dependent coefficients and 5 stages is constructed based on the 7th order method of Chan and Tsai. The new methods are applied to 4 well known oscillatory problems and their performance is compared with the methods in that of Chan and Tsai.The numerical experiments show the efficiency of the derived methods.
\end{abstract}

Keywords: Two-derivative Runge-Kutta methods; dispersion; dissipation; orbital problems

MSC: 65L05; 65L06

\section{Introduction}

We consider systems of first order ODEs of the form

$$
y^{\prime}(x)=f(x, y(x)), \quad x \in\left[x_{0}, X\right], \quad y\left(x_{0}\right)=y_{0}
$$

whose solutions have oscillatory or periodic behaviour. The well known Runge-Kutta (RK) methods are used to solve this problem for more than a century. Chan and Tsai [1] considered methods where only the first derivative of $f(x, y(x))$ (or second derivative of $y$ ) is involved; these methods are called Two-Derivative Runge-Kutta (TDRK) methods. The idea dates back to 1972 [2] where Kastlunger and Wanner introduced Runge-Kutta processes with multiple nodes. These methods use evaluations of $f(x, y(x))$ and its derivatives of $f(x, y(x))$ at the intermediate points. The advantage of TDRK methods is that they attain higher order with fewer stages than RK methods. Several authors considered numerical integration of ODEs using methods with properties such as high dispersive or dissipative order, phase-fitted, amplification-fitted, trigonometrically fitted methods, some recent articles are [3-23]. These methods have either constant or variable (frequency dependent) coefficients and are suitable for the numerical integration of systems with periodic or oscillatory behavior of the solution.

Recently TDRK methods with special properties have been considered in order to integrate ODEs with periodic or oscillatory behavior of the solution. These are methods with either constant or variable (frequency dependent) coefficients.

Fang et al. [24] present trigonometrically-fitting conditions for TDRK methods of the special type where the function $f$ is evaluated only once at each step and constructed Trigonometrically-Fitted (TFTDRK) methods. Considering the general case of TDRK methods that use several $f$ evaluations at each step the authors in [25] derived trigonometricallyfitting conditions generalizing the conditions given in [24]. Also considering the general 
case the authors in [26] present 5th order TDRK methods with constant coefficients and in [27] constructed modified TFTDRK methods.

Ahmad et al. [28] presented a phase-fitted and amplification-fitted TDRK method of higher order 6 with 4 stages. Fang et al. in [29] constructed two 6th order TDRK methods with 4 stages and increased phase-lag and dissipation order for the Schrödinger equation. The authors in [30] present 5th order TDRK methods with 3 and 4 stages with minimum dispersion and dissipation error. Also in [31] they present TDRK methods of order 6 with 4 stages.

In this work we construct special explicit TDRK methods of algebraic order 6 and 7, dispersion order 8 to 12, dissipation order 7 and 9 using 4,5 and 6 stages, as well as, a phase-fitted and amplification fitted method. In Section 2 we summarize some results on TDRK methods, Section 3 is devoted to the construction of the new methods. Numerical results are presented in Section 4 using four well known test problems.

\section{Background Theory}

\subsection{Two-Derivative Runge-Kutta Methods}

A TDRK method with s stages is of the form

$$
\begin{aligned}
Y_{i} & =y_{n}+h \sum_{j=1}^{s} a_{i j} f\left(x_{n}+c_{j} h, Y_{j}\right)+h^{2} \sum_{j=1}^{s} \hat{a}_{i j} g\left(x_{n}+c_{j} h, Y_{j}\right) \\
y_{n+1} & =y_{n}+h \sum_{i=1}^{s} b_{i} f\left(x_{n}+c_{i} h, Y_{i}\right)+h^{2} \sum_{i=1}^{s} \hat{b}_{i} g\left(x_{n}+c_{i} h, Y_{i}\right)
\end{aligned}
$$

where $y^{\prime \prime}(x, y(x))=g(x, y(x))=f_{x}(x, y(x))+f_{y}(x, y(x)) f(x, y(x))$, and $h$ is the step size. The associated Butcher tableau is

\begin{tabular}{c|c||c}
$c$ & $A$ & $\hat{A}$ \\
\hline & & $b^{T}$
\end{tabular}

where $A$ and $\hat{A}$ are square matrices of size $s, b$ and $\hat{b}, c$ are vectors of length $s$.

\subsection{Special TDRK Methods}

Chan and Tsai [1] considered explicit methods of a specific type where the function $f$ is evaluated only once at each step

$$
\begin{aligned}
Y_{i} & =y_{n}+h c_{i} f\left(x_{n}, y_{n}\right)+h^{2} \sum_{j=1}^{s} \hat{a}_{i j} g\left(x_{n}+c_{j} h, Y_{j}\right) \\
y_{n+1} & =y_{n}+h f\left(x_{n}, y_{n}\right)+h^{2} \sum_{i=1}^{s} \hat{b}_{i} g\left(x_{n}+c_{i} h, Y_{i}\right)
\end{aligned}
$$

the associated Butcher tableau is

$$
\begin{array}{l||l}
c & \hat{A} \\
\hline & \hat{b}^{T}
\end{array}
$$

where $c_{1}=0$ and $\hat{A}$ is strictly lower triangular with $\hat{a}_{i j}=0$ if $i \leq j$.

This is a method of the form (2) with $A$ and $b$

$$
\begin{aligned}
& a_{i 1}=c_{i}, a_{i j}=0 \text { for } j=2, \ldots, s, i=1, \ldots, s \\
& b_{1}=1, b_{i}=0 \text { for } i=2, \ldots, s
\end{aligned}
$$

Let $C$ be a diagonal matrix with diagonal the vector $c$ and $e=(1,1, \ldots, 1)$ a vector of size $s$. The order conditions for order up to seven are given in [1] based on Butcher's the 
algebraic theory of trees [32] (see also Hairer [33]). The order conditions for order 2, 3 and 4 are

$$
\hat{b}^{T} e=\frac{1}{2}, \hat{b}^{T} C e=\frac{1}{6}, \hat{b}^{T} C^{2} e=\frac{1}{12}
$$

the order conditions for orders 5, 6 and 7 are

$$
\begin{array}{ll}
\text { (order 5) } & \hat{b}^{T} C^{3} e=\frac{1}{20}, \quad \hat{b}^{T} \hat{A} C e=\frac{1}{120}, \\
\text { (order 6) } & \hat{b}^{T} C^{4} e=\frac{1}{30}, \quad \hat{b}^{T} C \hat{A} C e=\frac{1}{180}, \quad \hat{b}^{T} \hat{A} C^{2} e=\frac{1}{360}, \\
\text { (order 7) } & \hat{b}^{T} C^{5} e=\frac{1}{42}, \quad \hat{b}^{T} C^{2} \hat{A} C e=\frac{1}{252}, \quad \hat{b}^{T} C \hat{A} C^{2} e=\frac{1}{504}, \quad \hat{b}^{T} \hat{A} C^{3} e=\frac{1}{840}, \quad \hat{b}^{T} \hat{A}^{2} C^{2} e=\frac{1}{5040} .
\end{array}
$$

As in the case of RK methods simplifying assumptions for TDRK methods are

$$
A C^{k-1} e+(k-1) \hat{A} C^{k-2} e=\frac{C^{k} e}{k}
$$

The first is the row condition $A e=C e$, the second is $\hat{A} e=\left(C^{2} e\right) / 2$ and the third is $\hat{A} C e=\left(C^{3} e\right) / 6$.

\subsection{Stability, Dispersion and Dissipation}

The stability function of a TDRK method is

$$
R(z)=1+z b^{T}\left(I-z A-z^{2} \hat{A}\right)^{-1} e+z^{2} \hat{b}^{T}\left(I-z A-z^{2} \hat{A}\right)^{-1} e
$$

We follow the theoretical framework of Van der Houwen and Sommeijer [34] in order to analyze the behavior of numerical methods for oscillatory problems. In [34] the definitions of dispersion (or phase-lag) error $\phi(v)$ and the dissipation (or amplification) error $\alpha(v)$ are given via the stability on the imaginary axis.

$$
\phi(v)=v-\arg R(i v), \quad \alpha(v)=1-|R(i v)|
$$

where $v=w h$ and $w$ is the frequency of the specific problem.

The first is the error in the phase of the numerical solution and the second is the error in the numerical damping.

A TDRK method is said to have dispersion order $p$ and dissipation order $q$ if

$$
\phi(v)=c_{\phi} v^{p+1}+O\left(v^{p+3}\right) \text { and } \alpha(v)=c_{\alpha} v^{q+1}+O\left(v^{q+3}\right) .
$$

For explicit TDRK methods the real and the imaginary part of the stability function $R(i v)$ are polynomials in $v^{2}$ of degree $s$

$$
R(i v)=A_{s}\left(v^{2}\right)+i v B_{s}\left(v^{2}\right),
$$

therefore $\phi(v)$ and $\alpha(v)$ can be written as

$$
\phi(v)=v-\arctan \left(v \frac{B_{S}\left(v^{2}\right)}{A_{S}\left(v^{2}\right)}\right) \quad \text { and } \quad \alpha(v)=1-\sqrt{A_{S}^{2}\left(v^{2}\right)+v^{2} B_{S}^{2}\left(v^{2}\right)} .
$$

\section{Construction of the New Methods}

\subsection{Methods with 4 Stages}

Chan and Tsai [1] have shown that 6th order can be attained, they present 3 such methods. The authors in [31] presented two 6th order methods with 4 stages and optimized 
dispersion and dissipation order. Here we present again the construction of these methods and we shall use them later for the numerical experiments.

If we apply the second simplifying assumption (6) and solve for $\hat{a}_{21}, \hat{a}_{32}, \hat{a}_{43}$ the remaining unknowns are 10 the $c_{i} \mathrm{~s}$, the $\hat{b}_{i}$ s and $\hat{a}_{31}, \hat{a}_{41}, \hat{a}_{42}$.

We start by solving the linear system equations $\hat{b}^{T} C^{i} e=1 /((i+1)(i+2))$ for $i=$ $0,1,2,3$ for the $\hat{b}_{i}$

$$
\begin{aligned}
& \hat{b}_{1}=\frac{-3+c_{3}\left(5-10 c_{4}\right)+5 c_{4}+5 c_{2}\left(1-2 c_{4}+c_{3}\left(-2+6 c_{4}\right)\right)}{60 c_{2} c_{3} c_{4}}, \\
& \hat{b}_{2}=\frac{3-5 c_{4}+5 c_{3}\left(-1+2 c_{4}\right)}{60 c_{2}\left(c_{2}-c_{3}\right)\left(c_{2}-c_{4}\right)}, \quad \hat{b}_{3}=\frac{3-5 c_{4}+5 c_{2}\left(-1+2 c_{4}\right)}{60 c_{3}\left(c_{3}+c_{2}\right)\left(c_{3}-c_{4}\right)}, \quad \hat{b}_{4}=\frac{-3+c_{2}\left(5-10 c_{3}\right)+5 c_{3}}{60 c_{4}\left(c_{2}-c_{4}\right)\left(c_{4}-c_{3}\right)} .
\end{aligned}
$$

Then we solve the remaining 3 equations of order 6 for $\hat{a}_{31}, \hat{a}_{41}$ and $\hat{a}_{42}$.

Finally from $\hat{b}^{T} C^{4} e=1 / 30$ we derive $c_{2}$.

The coefficients $c_{3}$ and $c_{4}$ are used to increase the dispersion and the dissipation order. The following methods are derived, the first has phase-lag order 10 and dissipation order 7 , this method has been also given in [29] as a 5th order method. The second method has phase-lag order 8 and dissipation order 9 .
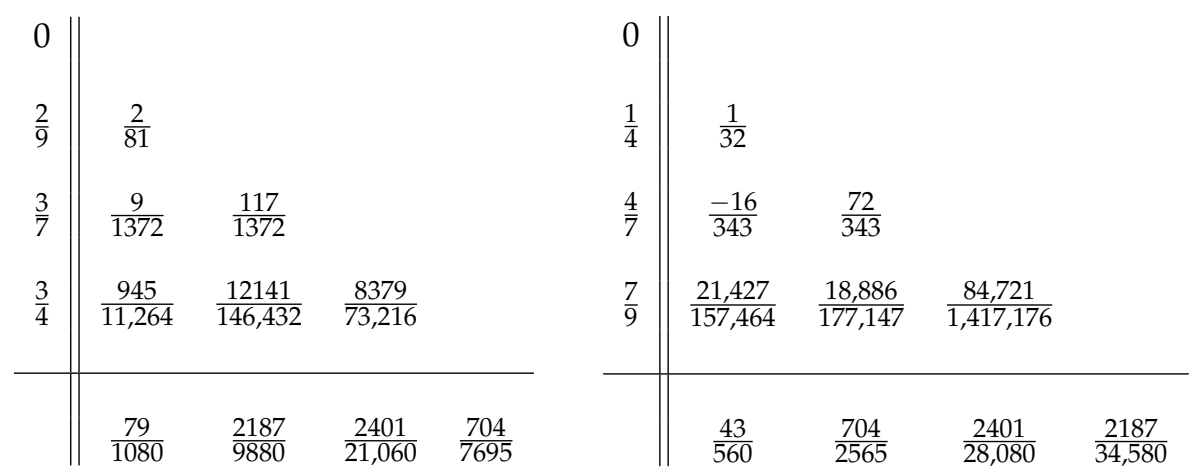

The phase-lag and the dissipation (8) of these method are

$$
\begin{aligned}
& \phi(v)=-\frac{v^{11}}{1,496,880}+O\left(v^{13}\right), \quad \alpha(v)=\frac{v^{8}}{362,880}+O\left(v^{10}\right) \\
& \phi(v)=\frac{v^{9}}{362,880}+O\left(v^{11}\right), \quad \alpha(v)=\frac{v^{10}}{403,200}+O\left(v^{12}\right)
\end{aligned}
$$

The stability functions (7) are

$$
R(z)=\sum_{k=0}^{7} \frac{z^{k}}{k !}+\frac{z^{8}}{40,320} \quad \text { and } \quad R(z)=\sum_{k=0}^{7} \frac{z^{k}}{k !}+\frac{z^{8}}{45,360}
$$

The methods presented in [1] are of 6th phase-lag order and 7th dissipation order:

$$
\begin{aligned}
& \phi(v)=-\frac{1}{22,680} v^{7}+O\left(v^{9}\right), \quad \alpha(v)=-\frac{1}{25,920} v^{8}+O\left(v^{10}\right) \\
& \phi(v)=-\frac{1}{5040} v^{7}+O\left(v^{9}\right), \quad \alpha(v)=-\frac{1}{5760} v^{8}+O\left(v^{10}\right)
\end{aligned}
$$

\subsection{Methods with 5 Stages}

\subsubsection{Method with Constant Coefficients}

Chan and Tsai in [1] presented two 7th order methods with 5 stages. In this section we also consider methods with 5 stages, and follow the next procedure to derive a method of order 7 . 
1. Using second simplifying assumption (6) we eliminate $\hat{a}_{i, i-1}$ for $i=2,3,4,5$.

2. Using third simplifying assumption (6) (except for the second component) we eliminate $\hat{a}_{i, i-2}$ for $i=3,4,5$. We have to set $\hat{b}_{2}=0$. Under this assumption the second condition for orders 5,6 and 7 follow from the first condition.

3. We solve the linear system equations

$$
\hat{b}^{T} C^{i} e=\frac{1}{(i+1)(i+2)}, \quad i=0,1,2,3
$$

for the $\hat{b}_{i}$ and derive

$$
\begin{aligned}
& \hat{b}_{1}=\frac{-3+c_{4}\left(5-10 c_{5}\right)+5 c_{5}+5 c_{3}\left(1-2 c_{5}+c_{4}\left(-2+6 c_{5}\right)\right)}{60 c_{3} c_{4} c_{5}}, \\
& \hat{b}_{3}=\frac{3-5 c_{5}+5 c_{4}\left(-1+2 c_{5}\right)}{60 c_{3}\left(c_{3}-c_{4}\right)\left(c_{3}-c_{5}\right)}, \quad \hat{b}_{4}=\frac{3-5 c_{5}+5 c_{3}\left(-1+2 c_{5}\right)}{60 c_{4}\left(c_{4}+c_{3}\right)\left(c_{4}-c_{5}\right)}, \quad \hat{b}_{5}=\frac{-3+c_{3}\left(5-10 c_{4}\right)+5 c_{4}}{60 c_{5}\left(c_{3}-c_{5}\right)\left(c_{5}+c_{4}\right)} .
\end{aligned}
$$

4. We solve the 3rd condition of order 6 for $\hat{a}_{41}$.

5. We solve the 3 rd and 4 th conditions of order 7 for $\hat{a}_{51}$ and $\hat{a}_{52}$.

6. We solve $\hat{b}^{T} C^{i} e=\frac{1}{(i+1)(i+2)}$ for $i=4,5$ and the last condition of order 7 for $c_{3}, c_{4}, c_{5}$.

7. Finally we determine $c_{2}$ to minimize the next term of the amplification error $c_{2}=(5+3 \sqrt{2}) / 28$

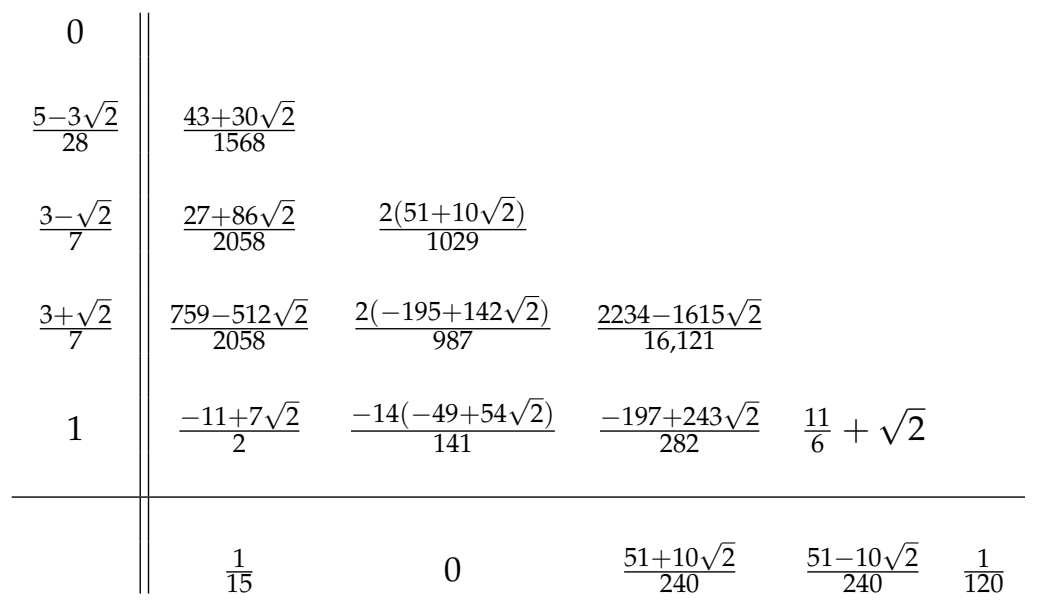

The stability function is

$$
R(z)=\sum_{k=0}^{8} \frac{z^{k}}{k !}+\frac{8+19 \sqrt{2}}{9,948,960} z^{9}+\frac{22+17 \sqrt{2}}{79,591,680} z^{10}
$$

the phase-lag and the dissipation are

$$
\phi(v)=\frac{(425+228 \sqrt{2}) v^{9}}{119,387,520}+O\left(v^{11}\right) \text { and } \alpha(v)=\frac{(133+75 \sqrt{2}) v^{10}}{44,217,600}+O\left(v^{12}\right)
$$




\subsubsection{Method with Variable Coefficients}

We also derive a phase-fitted and amplification-fitted method by modifying the first 7th order 5 stages method in [1]. Asking for $\phi(v)=0$ and $\alpha(v)=0$ we modify the coefficients $\hat{b}_{1}$ and $\hat{b}_{5}$ as follows

$$
\begin{aligned}
& \hat{b}_{1}=\frac{v^{3}\left(k_{1}+k_{2} \cos (v)+k_{3} \sin (v)\right)}{6720 d} \\
& \hat{b}_{5}=\frac{91\left(v\left(5400-848 v^{2}+35 v^{4}\right)-5400 \sin (v)\right)}{675 d}
\end{aligned}
$$

where

$$
\begin{aligned}
& d=v^{3}\left(728-140 v^{2}+15 v^{4}\right) \\
& k_{1}=\left(236,376+21,812 v^{2}-13,985 v^{4}+800 v^{6}\right) \\
& k_{2}=-6720 v\left(728-140 v^{2}+15 v^{4}\right) \\
& k_{3}=-960\left(-5096+2548 v^{2}-245 v^{4}+15 v^{6}\right)
\end{aligned}
$$

The Taylor expansions of the coefficients $\hat{b}_{1}$ and $\hat{b}_{5}$ are

$$
\begin{aligned}
& \hat{b}_{1}=\frac{71}{960}+\frac{v^{6}}{158,760}-\frac{31 v^{8}}{51,891,840}-\frac{107 v^{10}}{1,311,710,400}-\frac{323,129 v^{12}}{48,343,086,792,000}+\frac{29,534,363 v^{14}}{68,376,461,958,604,800}+O\left(h^{16}\right) \\
& \hat{b}_{5}=\frac{13}{1350}-\frac{v^{6}}{362,880}-\frac{131 v^{8}}{259,459,200}-\frac{2867 v^{10}}{70,832,361,600}+\frac{82,717 v^{12}}{1,570,995,456,000}+\frac{7,318,085,461 v^{14}}{5,470,116,956,688,384,000}+O\left(h^{16}\right)
\end{aligned}
$$

The order conditions now become

$$
\begin{array}{ll}
\hat{b}^{T} e=\frac{1}{2}+\frac{v^{6}}{282,240}+O\left(v^{8}\right) & \hat{b}^{T} C^{5} e=\frac{1}{42}-\frac{v^{6}}{362,880}+O\left(v^{8}\right) \\
\hat{b}^{T} C e=\frac{1}{6}-\frac{v^{6}}{362,880}+O\left(v^{8}\right) & \hat{b}^{T} C^{2} \hat{A} C e=\frac{1}{252}-\frac{v^{6}}{1,886,976}+O\left(v^{8}\right) \\
\hat{b}^{T} C^{2} e=\frac{1}{12}-\frac{v^{6}}{362,880}+O\left(v^{8}\right) & \hat{b}^{T} C \hat{A} C^{2} e=\frac{1}{504}-\frac{v^{6}}{3,773,952}+O\left(v^{8}\right) \\
\hat{b}^{T} C^{3} e=\frac{1}{20}-\frac{v^{6}}{362,880}+O\left(v^{8}\right) & \hat{b}^{T} \hat{A} C^{3} e=\frac{1}{840}-\frac{5 v^{6}}{26,417,664}+O\left(v^{8}\right) \\
\hat{b}^{T} \hat{A} C e=\frac{1}{120}-\frac{v^{6}}{1,886,976}+O\left(v^{8}\right) & \hat{b}^{T} \hat{A}^{2} C e=\frac{1}{5040}-\frac{v^{6}}{17,611,776}+O\left(v^{8}\right) \\
\hat{b}^{T} C^{4} e=\frac{1}{30}-\frac{v^{6}}{362,880}+O\left(v^{8}\right) & \\
\hat{b}^{T} C \hat{A} C e=\frac{1}{180}-\frac{v^{6}}{1,886,976}+O\left(v^{8}\right) & \\
\hat{b}^{T} \hat{A} C^{2} e=\frac{1}{360}-\frac{v^{6}}{3,773,952}+O\left(v^{8}\right) &
\end{array}
$$

We see that the modified method reduces to the original method as $v \rightarrow 0$.

\subsection{Methods with 6 Stages}

As we have seen in the construction of the 7 th order method using 5 stages there is only one free parameter $c_{2}$ which we determine by nullifying the next term of the amplification error. In order to have more flexibility for further optimization of the method we consider 6 stages. We impose the first two simplifying assumptions and follow the procedure:

1. Using second simplifying assumption eliminate $\hat{a}_{i, i-1}$ for $i=2,3,4,5,6$.

2. Choose $c_{2}=0.2, c_{3}=0.4, c_{4}=0.6, c_{5}=0.8, c_{6}=1$. 
3. Solve the linear system equations

$$
\hat{b}^{T} C^{i} e=\frac{1}{(i+1)(i+2)}, \quad i=0,1, \ldots, 5
$$

for the $\hat{b}_{i}$ for $i=1, \ldots, 6$.

4. Solve the remaining conditions for $\hat{a}_{i, j}, i=3,4,5, j=1, \ldots, i-2$ and $\hat{a}_{64}$.

The phase-lag order of this method is 8 and the dissipation order is 7 . We find the remaining coefficients $\hat{a}_{61}, \hat{a}_{62}, \hat{a}_{63}$ requiring the method to have phase-lag and dissipation order 12 and 9 . The resulting method is:

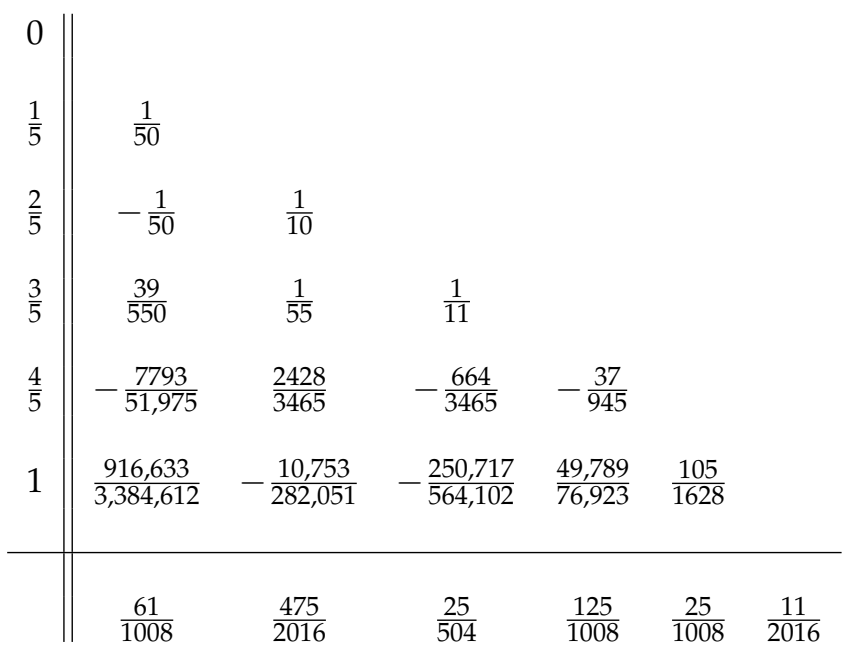

The stability function is

$$
R(z)=\sum_{k=0}^{9} \frac{z^{k}}{k !}+\frac{z^{10}}{4,435,200}+\frac{z^{11}}{39,916,800}+\frac{z^{12}}{399,168,000}
$$

the phase-lag and the dissipation are

$$
\phi(v)=-\frac{191}{15,567,552,000} v^{13}+O\left(v^{15}\right), \quad \alpha(v)=-\frac{1}{19,958,400} v^{10}+O\left(v^{14}\right) .
$$

\section{Numerical Results}

The methods used to illustrate the efficiency of the new method are

- CTs4o6 the 6th order method with 4 stages in [1] (second method)

- $\quad$ CTs5o7 the 7th order method with 5 stages in [1] (first method)

- $\quad$ NEWs4o6B the new optimized 6th order method with 4 stages in Section 3.1 (second method)

- $\quad$ NEWs5o7 the new optimized 7th order method with 5 stages in Section 3.2.1

- $\quad$ NEWs6o7 the new optimized 7th order method with 6 stages in Section 3.3

- $\quad$ PFs5 the new phase-fitted and amplification-fitted method 5 stages in Section 3.2.2

- $\quad$ RKNs4 the 5th order Runge-Kutta-Nyström method with 4 stages ([33], p. 285)

- $\quad$ optRKNs4 the optimized Runge-Kutta-Nyström method with 4 stages, algebraic order 4 and phase-lag order 8 ([35]).

The problems used are

- The harmonic oscillator

- The inhomogeneous oscillatory problem that can be found in [36]

- The oscillatory linear system studied by Franco in [37]

- The almost periodic orbit problem studied by Stiefel and Bettis [38] 


\subsection{Problem 1}

The harmonic oscillator

$$
\begin{array}{lll}
y_{1}^{\prime \prime}=-y_{1}, & y_{1}(0)=1, & y_{1}^{\prime}(0)=0 \\
y_{2}^{\prime \prime}=-y_{2}, & y_{2}(0)=0, & y_{2}^{\prime}(0)=1
\end{array}
$$

The exact solution is

$$
y_{1}(x)=\cos (x) \quad y_{2}(x)=\sin (x)
$$

In Figure 1 we present the maximum absolute error of the solution in the interval $[0,1000]$. For this problem we use $w=1$. The fitted method has maximum absolute error less than $10^{-14}$ and for this reason it is not in the figure. The 3 methods with minimized phase-lag and amplification error have clearly superior performance compared with the methods in [1].

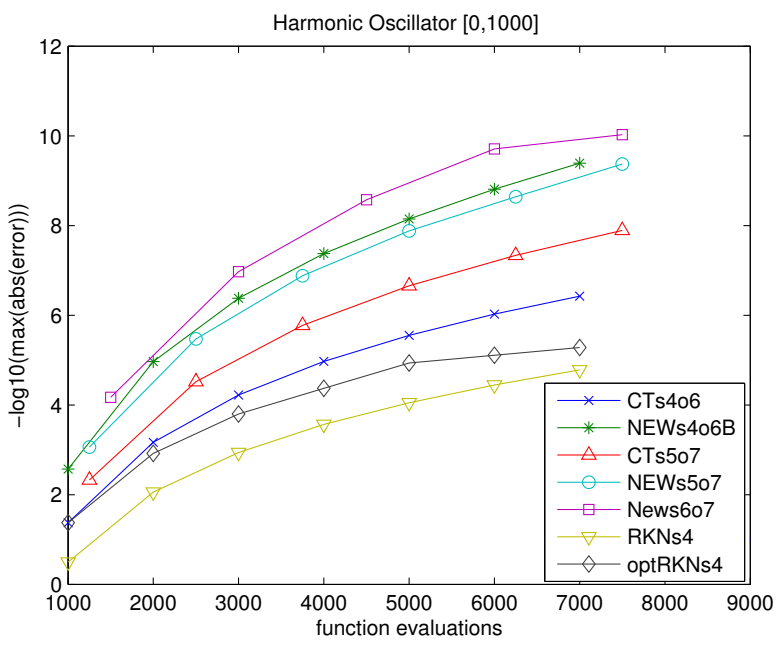

Figure 1. Problem 1 (Harmonic Oscillator) [0, 1000].

\subsection{Problem 2}

The inhomogeneous oscillatory problem

$$
y^{\prime \prime}=-100 y+99 \sin (x), \quad y(0)=1, \quad y^{\prime}(0)=11 .
$$

The exact solution is $y(x)=\cos (10 x)+\sin (10 x)+\sin (x)$. For this problem we use $w=10$. In Figure 2 we present the maximum absolute error of the solution in the interval $[0,100]$. Results again show the high accuracy of the fitted method, performance of all methods tested is similar to that for Problem 1.

\subsection{Problem 3}

The oscillatory linear system

$$
\begin{aligned}
& y_{1}^{\prime \prime}+13 y_{1}-12 y_{2}=9 \cos (2 x)-12 \sin (2 x), \quad y_{1}(0)=1, \quad y_{1}^{\prime}(0)=-4 \\
& y_{2}^{\prime \prime}-12 y_{1}+13 y_{2}=-12 \cos (2 x)+9 \sin (2 x), \quad y_{2}(0)=0, \quad y_{2}^{\prime}(0)=8 .
\end{aligned}
$$

The exact solution is

$$
\begin{aligned}
& y_{1}(x)=\sin (x)-\sin (5 x)+\cos (2 x) \\
& y_{2}(x)=\sin (x)+\sin (5 x)+\sin (2 x)
\end{aligned}
$$


For this problem we use $w=5$. In Figure 3 we present the maximum absolute error of the solution in the interval $[0,100]$, we see that the new methods produce less absolute error.

\subsection{Problem 4}

The almost periodic orbit problem studied by Stiefel and Bettis [38]:

$$
\begin{array}{lll}
y_{1}^{\prime \prime}=-y_{1}+0.001 \cos (x), & y_{1}(0)=1, & y_{1}^{\prime}(0)=0 \\
y_{2}^{\prime \prime}=-y_{2}+0.001 \sin (x), & y_{2}(0)=0, & y_{2}^{\prime}(0)=0.9995
\end{array}
$$

The exact solution is

$$
y_{1}(x)=\cos (x)+0.0005 x \sin (x), \quad y_{2}(x)=\sin (x)-0.0005 x \cos (x) .
$$

For this problem we use $w=1$. In Figure 4 we present the maximum absolute error of the solution in the interval $[0,1000]$.

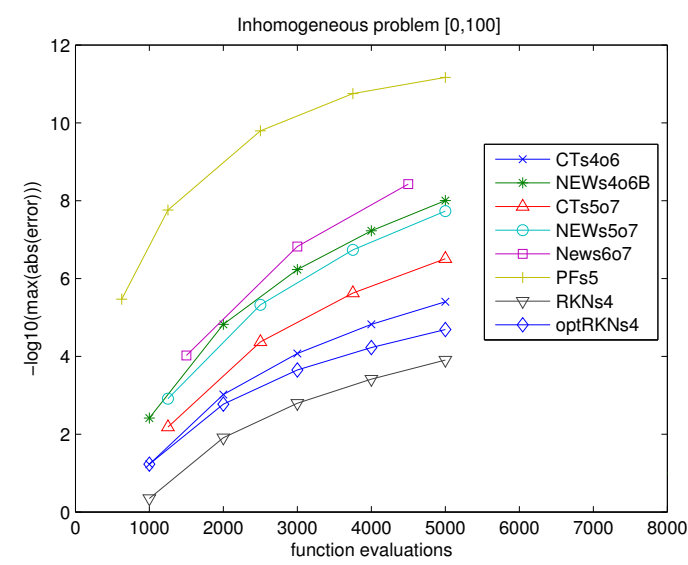

Figure 2. Problem 2 (Inhomogeneous Oscillatory Problem) [0, 100].

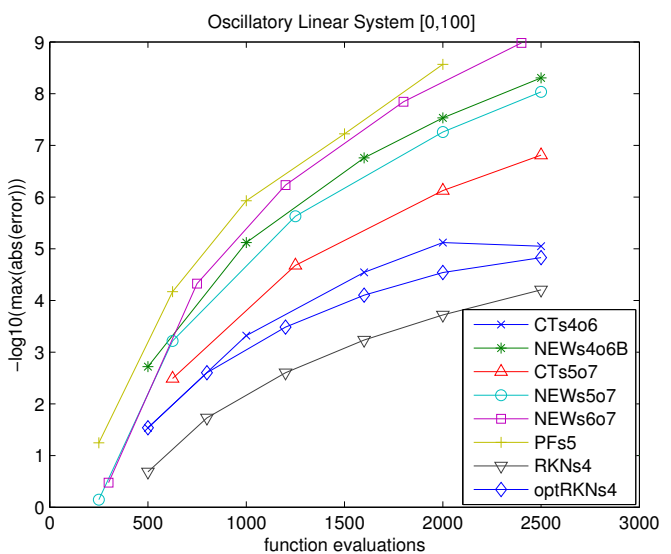

Figure 3. Problem 3 (Oscillatory Linear System) [0, 100]. 


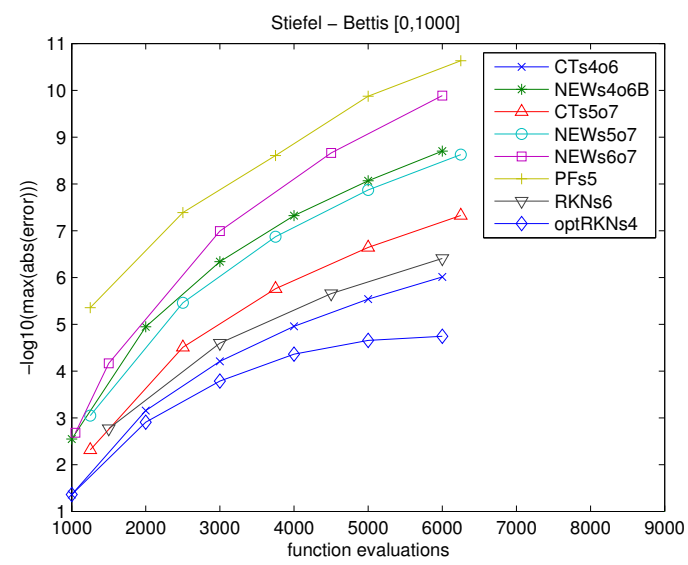

Figure 4. Problem 4 (Stiefel-Bettis) [0, 1000].

\section{Conclusions}

In this work two new methods are presented of order 7 with 5 and 6 stages and minimized dispersion and dissipation error, as well as a modified phase-fitted, amplificationfitted method with frequency dependent coefficients and 5 stages. The new methods have been tested on 4 oscillatory problems. The numerical results show that the new methods attain higher accuracy at the same cost.

Author Contributions: T.M., Z.K. contributed equally to this work. All authors have read and agreed to the published version of the manuscript.

Funding: This research received no external funding.

Institutional Review Board Statement: Not applicable.

Informed Consent Statement: Not applicable.

Data Availability Statement: Not applicable.

Conflicts of Interest: The authors declare no conflict of interest.

\section{References}

1. Chan, R.P.K.; Tsai, A.Y.J. On explicit two-derivative Runge-Kutta methods. Numer. Alg. 2010, 53, 171-194. [CrossRef]

2. Kastlunger, K.H.; Wanner, G. Runge Kutta Processes with Multiple nodes. Computing 1972, 9, 9-24. [CrossRef]

3. Vigo-Aguiar, J.; Martín-Vaquero, J.; Ramos, H. Exponential fitting BDF-Runge-Kutta algorithms. Comput. Phys. Commun. 2018, 178, 15-34. [CrossRef]

4. Ramos, H.; Vigo-Aguiar, J. On the frequency choice in trigonometrically fitted methods. Appl. Math. Lett. 2010, 23, 1378-1381. [CrossRef]

5. Vigo-Aguiar, J.; Ramos, H. On the choice of the frequency in trigonometrically-fitted methods for periodic problems. J. Comput. Appl. Math. 2015, 277, 94-105. [CrossRef]

6. Ramos, H.; Kalogiratou, Z.; Monovasilis, T.; Simos, T.E. An optimized two-step hybrid block method for solving general second order initial-value problems. Numer. Algor. 2016, 72, 1089-1102. [CrossRef]

7. Simos, T.E.; Tsitouras, C. Explicit, ninth order, two step methods for solving inhomogeneous linear problems $x(t)=\Lambda x(t)+f(t)$. Appl. Numer. Math. 2020, 153, 344-351. [CrossRef]

8. Kovalnogov, V.N.; Simos, T.E.; Tsitouras, C. Ninth-order, explicit, two-step methods for second order inhomogeneous linear IVPs. Math. Meth. Appl. Sci. 2020, 43, 4918-4926. [CrossRef]

9. Alolyan, I.; Simos, T.E.; Tsitouras, C. Eighth-order, phase-fitted, four-step methods for solving y =f (x, y). Math. Meth. Appl. Sci. 2020, 43, 4016-4022. [CrossRef]

10. Hou, C.-C.; Simos, T.E.; Famelis, I.T. Neural network solution of pantograph type differential equations. Math. Meth. Appl. Sci. 2020, 43, 3369-3374. [CrossRef]

11. Medvedev, M.A.; Simos, T.E.; Tsitouras, C. Low-order, P-stable, two-step methods for use with lax accuracies. Math. Meth. Appl. Sci. 2019, 42, 6301-6314. [CrossRef]

12. Medvedeva, M.A.; Simos, T.E.; Tsitouras, C. Trigonometric fitted modification of RADAU5. Math. Meth. Appl. Sci. 2020, 43, 1582-1589. [CrossRef] 
13. Lin, C.; Hsu, C.W.; Simos, T.E.; Tsitouras, C. Explicit, semi-symmetric, hybrid, six-step, eighth order methods for solving $\mathrm{y}=\mathrm{f}(\mathrm{x}, \mathrm{y})$. Appl. Comput. Math. 2019, 18, 296-304.

14. Medvedeva, M.A.; Simos, T.E.; Tsitouras, C. Variable step-size implementation of the sixth-order Numerov-type methods. Math. Meth. Appl. Sci. 2020, 43, 1204-1215. [CrossRef]

15. Alolyan, I.; Simos, T.E.; Tsitouras, C. Interpolants for sixth-order Numerov-type methods. Math. Meth. Appl. Sci. 2019, 42, 7349-7358. [CrossRef]

16. Medvedev, M.A.; Simos, T.E.; Tsitouras, C. Local interpolants for Numerov-type methods and their implementation in variable step schemes. Math. Meth. Appl. Sci. 2019, 42, 7047-7058. [CrossRef]

17. Fang, J.; Liu, C.; Simos, T.E.; Famelis, I.T. Neural network solution of single delay differential equations. Mediterr. J. Math. 2020 17, 30. [CrossRef]

18. Liu, C.; Hsu, C.-W.; Simos, T.E.; Tsitouras, C. Phase-fitted, six-step methods for solving x = f (t, x). Math. Meth. Appl. Sci. 2019, 42, 3942-3949. [CrossRef]

19. Liu, C.; Hsu, C.-W.; Tsitouras, C.; Simos, T.E. Hybrid Numerov-type methods with coefficients trained to perform better on classical orbits. Bull. Malays. Math. Sci. Soc. 2019, 42, 2119-2134. [CrossRef]

20. Lin, C.; Chen, J.J.; Simos, T.E.; Tsitouras, C. Evolutionary derivation of sixth-order P-stable SDIRKN methods for the solution of PDEs with the method of lines. Mediterr. J. Math. 2019, 16, 69. [CrossRef]

21. Fang, J.; Liu, C.; Hsu, C.-W.; Simos, T.E.; Tsitouras, C. Explicit hybrid six-step, sixth order, fully symmetric methods for solving y =f (x, y). Math. Meth. Appl. Sci. 2019, 42 , 3305-3314. [CrossRef]

22. Medvedev, M.A.; Simos, T.E.; Tsitouras, C. Hybrid, phase-fitted, four-step methods of seventh order for solving $x(t)=\mathrm{f}(\mathrm{t}, \mathrm{x})$. Math. Meth. Appl. Sci. 2019, 42, 2025-2032. [CrossRef]

23. Medvedev, M.A.; Simos, T.E.; Tsitouras, C. Trigonometric-fitted hybrid four-step methods of sixth order for solving $\mathrm{y}(\mathrm{x})=\mathrm{f}(\mathrm{x}, \mathrm{y})$ Math. Meth. Appl. Sci. 2019, $42,710-716$. [CrossRef]

24. Fang, Y.; You, X.; Ming, Q. Trigonometrically fitted two-derivative Runge-Kutta methods for solving oscillatory differential equations. Numer. Algor. 2014 65, 651-667. [CrossRef]

25. Monovasilis, T.; Kalogiratou, Z.; Simos, T.E. Trigonometrical fitting conditions for two derivative Runge Kutta methods Numer. Algor. 2018, 79, 787-800. [CrossRef]

26. Kalogiratou, Z.; Monovasilis, T.; Simos, T.E. Construction of two derivative Runge Kutta methods of order five. AIP Conf. Proc. 2017, 1863, 560092 .

27. Kalogiratou, Z.; Monovasilis, T.; Simos, T.E. New fifth-order two-derivative Runge-Kutta methods with constant and frequencydependent coefficients. Math. Meth. Appl. Sci. 2019, 42, 1955-1966. [CrossRef]

28. Ahmad, N.A.; Senu, N.; Ismail, F. Phase-Fitted and Amplification-Fitted Higher Order Two-Derivative Runge-Kutta Method for the Numerical Solution of Orbital and Related Periodical IVPs. Math. Probl. Eng. 2017, 2017, 1871278. [CrossRef]

29. Fang, Y.; Zhang, Y.; Wang, P. Two-derivative Runge-Kutta methods with increased phase-lag and dissipation order for the Schrödinger equation. J. Math. Chem. 2018, 56, 1924-1934. [CrossRef]

30. Kalogiratou, Z.; Monovasilis, T.; Simos, T.E. Two-derivative Runge-Kutta methods with optimal phase properties. Math. Meth. Appl. Sci. 2020, 43, 1267-1277. [CrossRef]

31. Monovasilis, T.; Kalogiratou, Z.; Simos, T.E. Optimized two derivative Runge-Kutta methods for solving orbital and oscillatory problems. AIP Conf. Proc. 2019, 2116, 450107.

32. Butcher, J.C. The Numerical Analysis of Ordinary Differential Equations; John Wiley Sons: Hoboken, NJ, USA, 1987.

33. Hairer, E.; Norsett, S.; Wanner, G. Solving Ordinary Differential Equations I: Nonstiff Problems; Springer: Berlin/Heidelberg, Germany, 2002.

34. Houwen, P.J.V.; Sommeijer, B.P. Explicit Runge-Kutta (-Nyström) methods with reduced phase errors for computing oscillating solutions. SIAM J. Numer. 1987, 24, 595-617. [CrossRef]

35. Simos, T.E.; Dimas, E.; Sideridis, A.B. A Runge-Kutta-Nyström method for the numerical integration of special second-order periodic initial-value problems. J. Comput. Appl. Math. 1994, 51, 317-326. [CrossRef]

36. Vigo-Aguiar, J.; Ramos, H. Dissipative Chebyshev exponential-fitted methods for numerical solution of second-order differential equations. J. Comput. Appl. Math. 2003, 158, 187-211. [CrossRef]

37. Franco, J.M. A class of explicit two-step hybrid methods for second-order IVPs. J. Comput. Appl. Math. 2006, 187, 41-57. [CrossRef]

38. Stiefel, E.; Bettis, D.G. Stabilization of Cowell's method. Numer. Math. 1969, 13, 154-175. [CrossRef] 